Irish Math. Soc. Bulletin

Number 76, Winter 2015, 77-78

ISSN 0791-5578

\title{
Boolean Rings are Definitely Commutative!
}

\author{
DESMOND MACHALE
}

Abstract. A ring $\{R,+,$.$\} is called Boolean if r^{2}=r$ for all $r \in R$. We present four proofs that a Boolean ring is commutative.

A ring $\{R,+,$.$\} is called Boolean if r^{2}=r$ for all $r \in R$. In this bicentenary year of Boole's birth we present four proofs that a Boolean ring is commutative. Our first proof is the standard one found in many textbooks.

Proof 1. For all $r \in R$ we have $r=r^{2}=(-r)^{2}=-r$, so $r+r=0$. Next, for all $x$ and $y$ in $R, x+y=(x+y)^{2}=x^{2}+x y+y x+y^{2}$, so by cancellation in the group $\{R,+\}$, we have $x y+y x=0=x y+x y$, by the above. Again by cancellation we have $x y=y x$, as required.

Proof 2. As in Proof 1, $x y+y x=0$, for all $x$ and $y$ in $R$. Since for all $r \in R, 0 . r=0=r .0$ we have $(x y+y x) x=x(x y+y x)$ or $x y x+y \cdot x^{2}=x^{2} \cdot y+x y x$. Cancelling $x y x$ and remembering that $x^{2}=x$, we get $x y=y x$, as required.

Proof 3. Since for all $r, r^{2}=r$ it follows that if $r^{2}=0$ then $r=0$. Now for all $x$ and $y$ in $R$ we have $(x y-x y x)^{2}=x y x y+x y x x y x-$ $x y x y x-x y x x y=x y x y+x y x y x-x y x y x-x y x y=0$. So $x y-x y x=0$ and $x y=x y x$. Then $(y x-x y x)^{2}=y x y x+x y x x y x-y x x y x-$ $x y x y x=y x y x+x y x y x-y x y x-x y x y x=0$. So $y x-x y x=0$ and $y x=x y x$. Thus $x y=y x$ as required.

Proof 4. For $a, b \in R$ if $a b=0$, then $b a=(b a)^{2}=b(a b) a=0$. Now, $0=x y-x y=x y-x^{2} y=x(y-x y)$, so $0=(y-x y) x=y x-x y x$. Also, $0=y x-y x=y x-y x^{2}=(y-y x) x$, so $0=x(y-y x)=$ $x y-x y x$. Thus $x y=y x$ for all $x$ and $y$ in $R$.

We note it is immediate in all four proofs that $x y=y x=x y x=$ $y x y$, for all $x$ and $y$.

2010 Mathematics Subject Classification. 19E50.

Key words and phrases. Boolean Rings.

Received on 8-6-2015. 
Desmond MacHale received his Ph.D. from the University of Keele and is Emeritus Professor of Mathematics at University College Cork where he taught for nearly forty years. His research interests include commutativity in groups and rings, automorphisms of groups, Euclidean geometry, number theory, and mathematical humour.

School of Mathematical Sciences, University College Cork.

E-mail address: d.machale@ucc.ie 